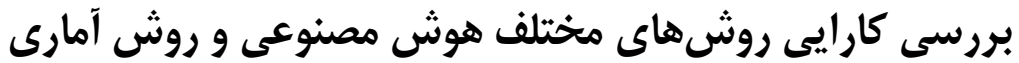

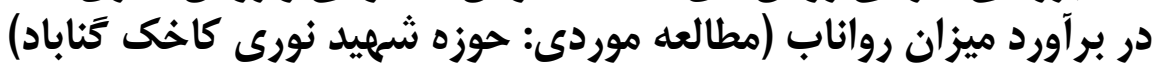

\author{
محمدمهردى زرعى '، محمدتقى دستور انى '، منصور مصداقى "و مسعود عشقى زادهء

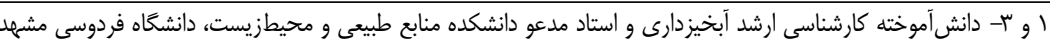

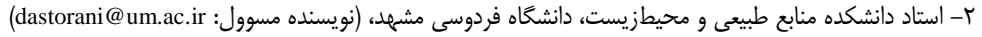

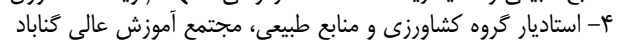

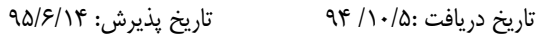

\begin{abstract}
جكيده

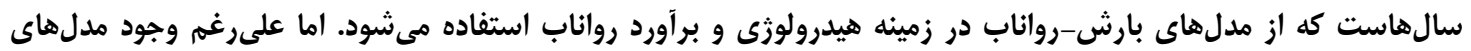

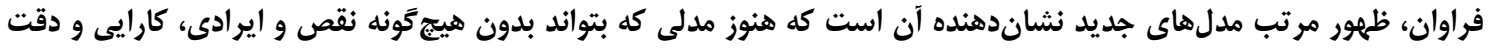

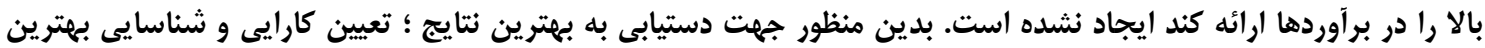

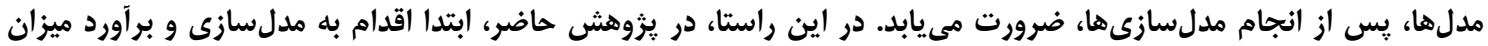

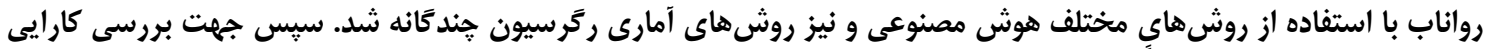

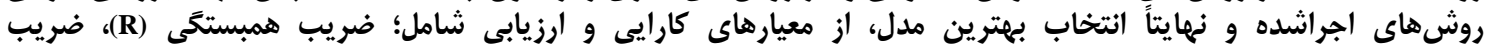

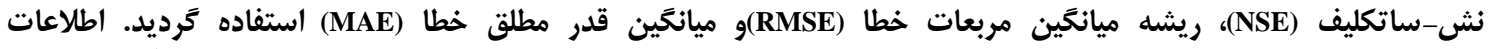

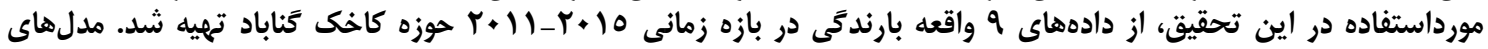

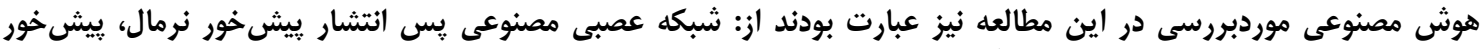

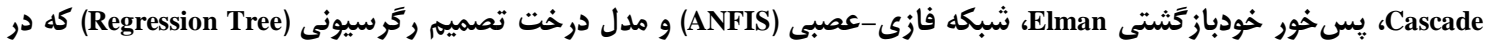

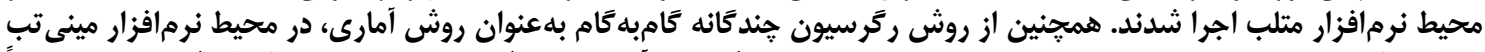

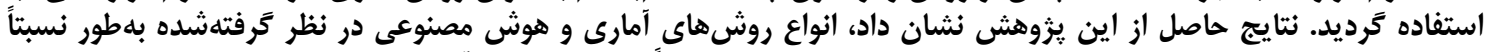

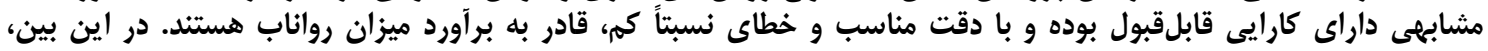

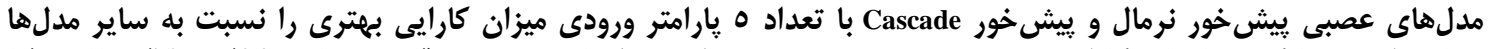

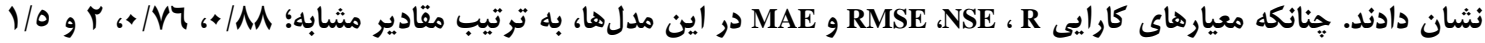

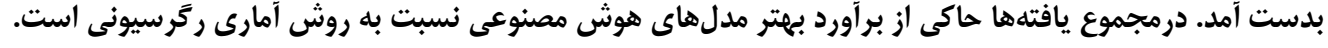

وازههاى كليدى: مدلسازى ، شبكههاى عصبى، درخت تصميم، ANFIS، پس انتشار، ييشخور، يسخور

معيارهاى ارزيابى، مناسبترين و كاراترين مدل را با توجه به به مايه

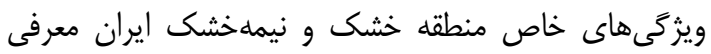

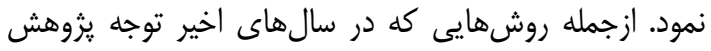

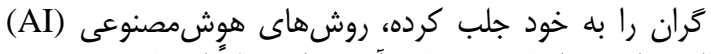

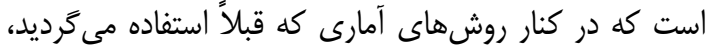



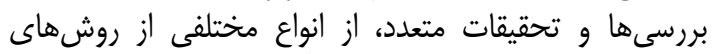

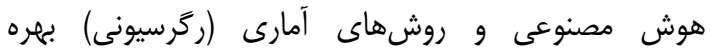

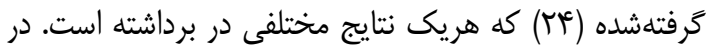

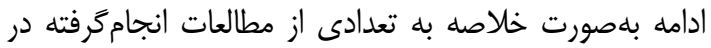

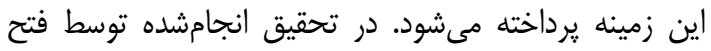

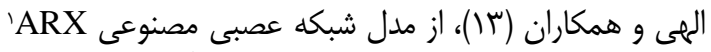

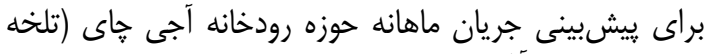

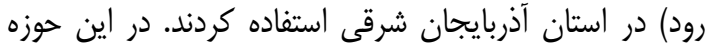

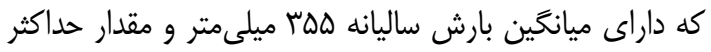

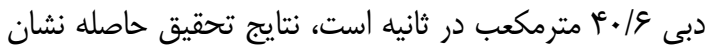

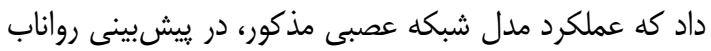

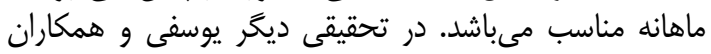

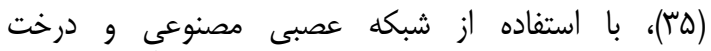

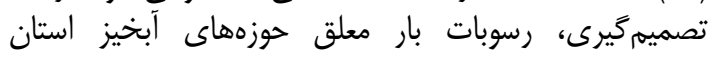

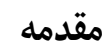

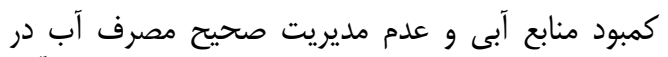

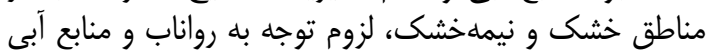

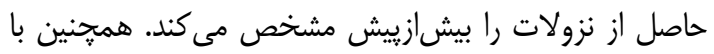

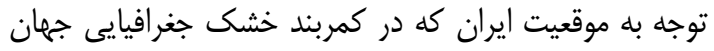

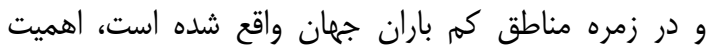

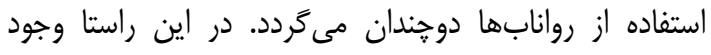

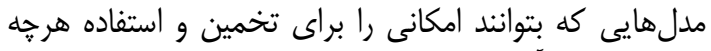

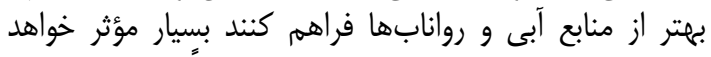

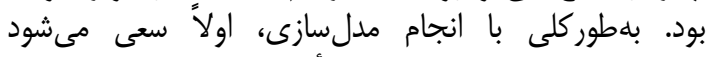

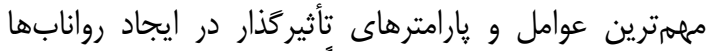

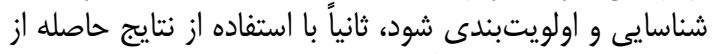

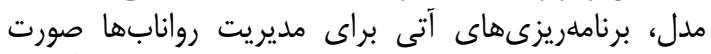

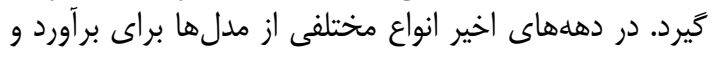

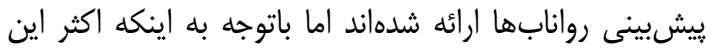

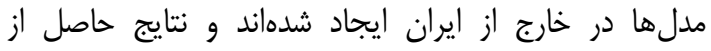

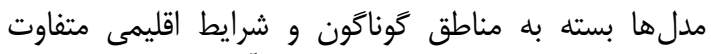

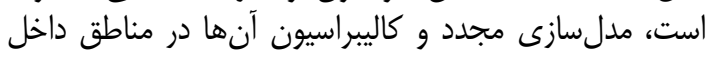

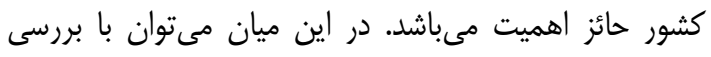

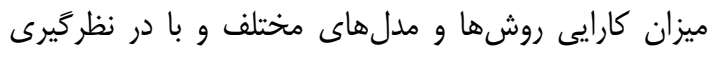


عملكرد را در بيشيينى دادههاى تست سه حوزه مذكور داشته إنها

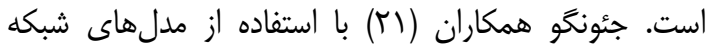

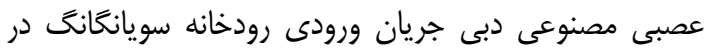

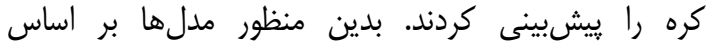

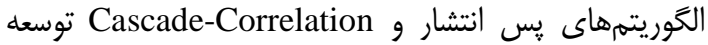
داده شد. نتايج نشان داد مدل Cascade-Correlation نسبت نداريت

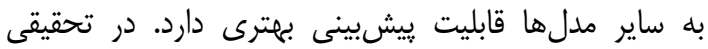

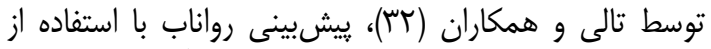

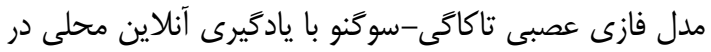

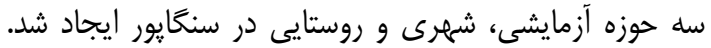

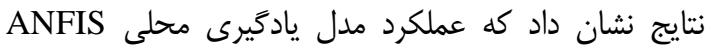

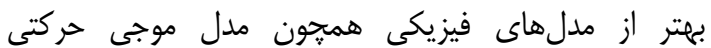

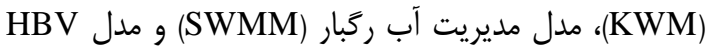

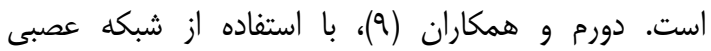

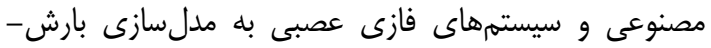
رواناب در حوزه آبخيز Susurluk يرداختند. نتايج نشان ندان دادي

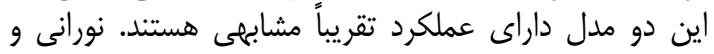

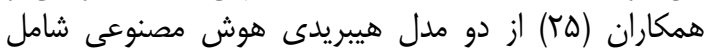

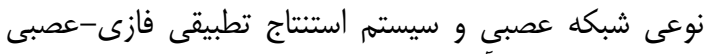

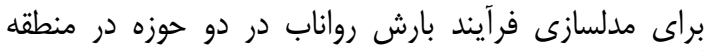

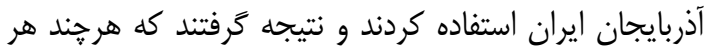



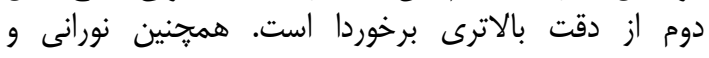

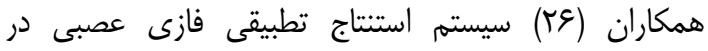

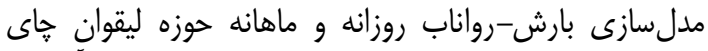

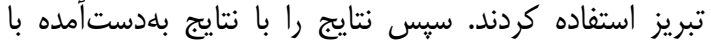

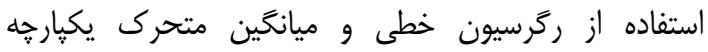
يسرونده (ARIMA) مقايسه كردند. درنهايت ايشان نتيجه

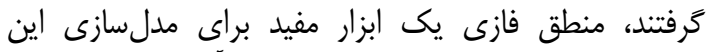

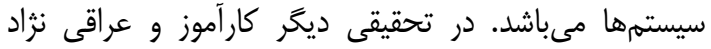

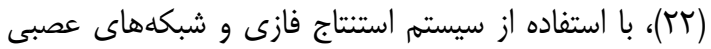

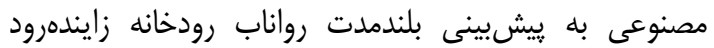

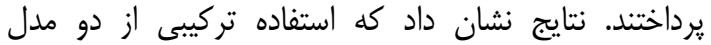

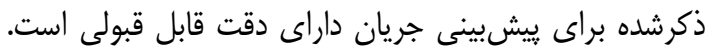

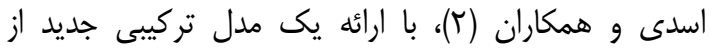

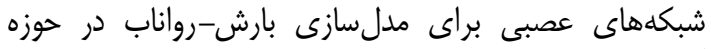

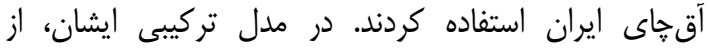

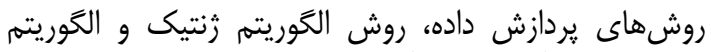

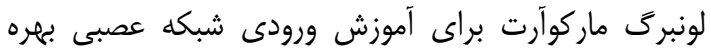

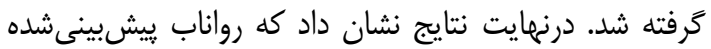

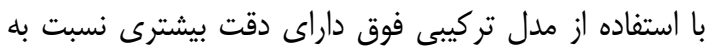

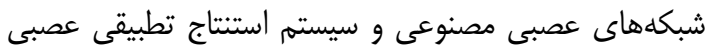

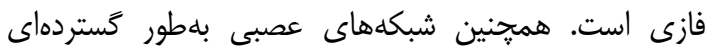

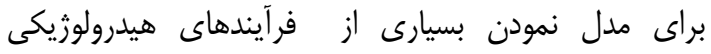

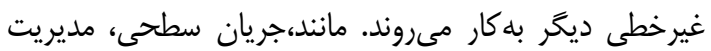

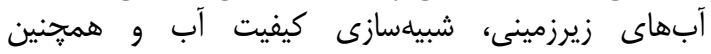

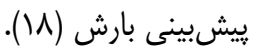

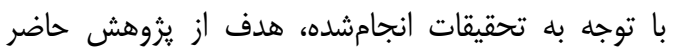

بررسى كارايى مدلهاى مختلف هوشمصنوعى و روشهات انداى
لرستان را شبيهسازى نمودند. نتايج نشان داد كه الكوريته

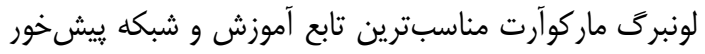

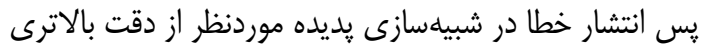

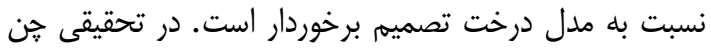

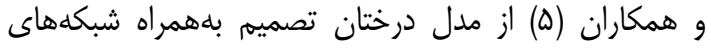

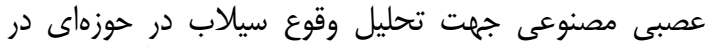

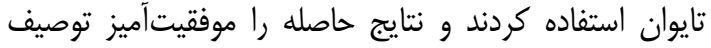

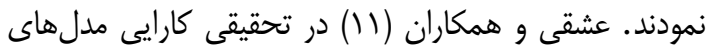

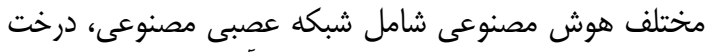

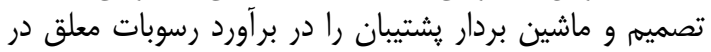
حوزه آبخيز بابا امان خراسان شمالى مورد ارزيابيى قرار دارديان دادند.



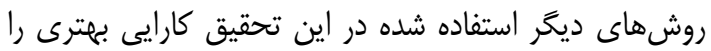

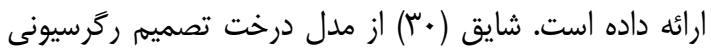



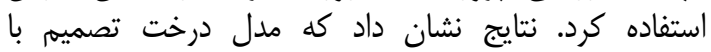

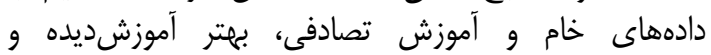

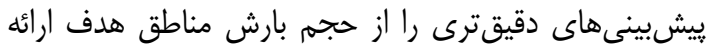

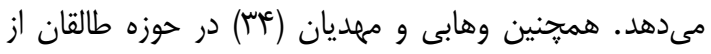

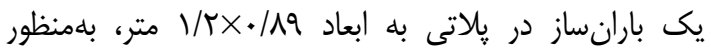

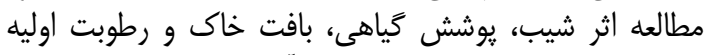

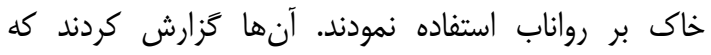

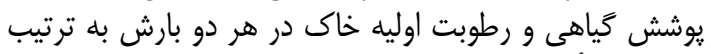

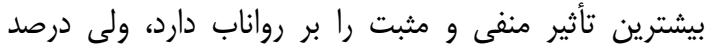

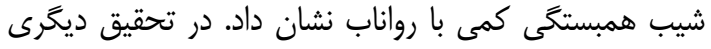

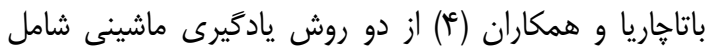

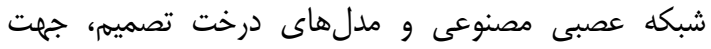

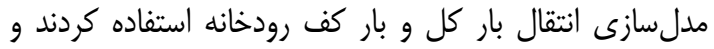

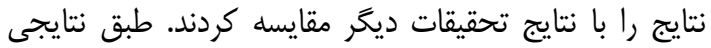

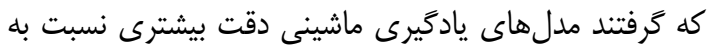

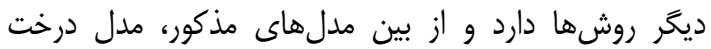

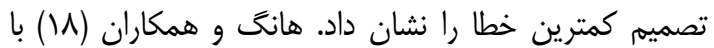
استفاده از دادههاى رواناب VD ايستخاه اطراف بان بانكوى تايلناند،



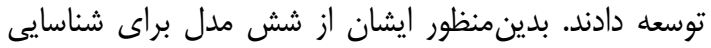

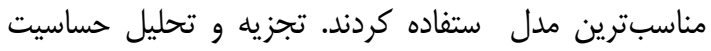

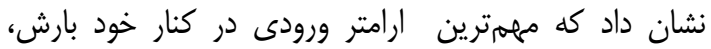

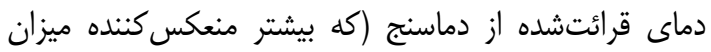

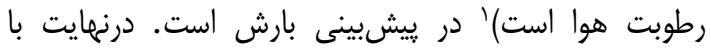



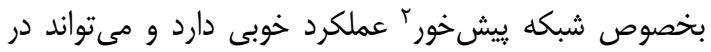

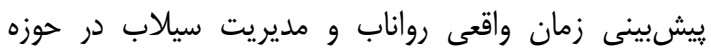

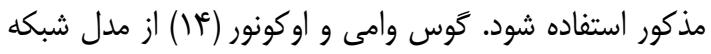

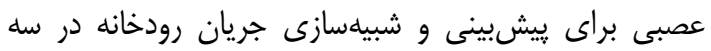

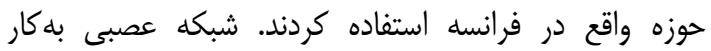

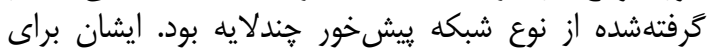



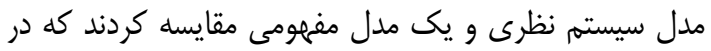
نهايت نتايج تحقيق نشان داد مدل شبكه مفري عصبى بهترين

2- Feed forward 


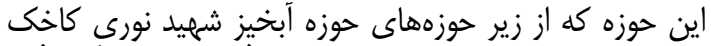

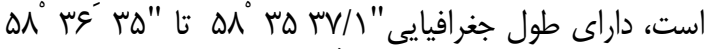

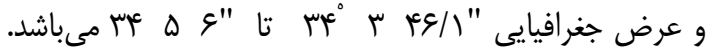

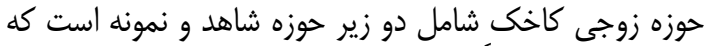

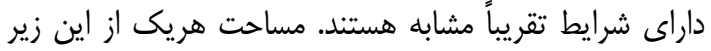

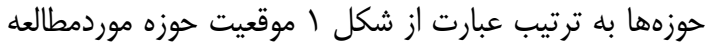

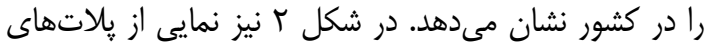

استفاده شده در تحقيق ديده مى شود (r) (I)
آمارى رَرسيونى در ييشبينى و برآورد ميزان رواناب

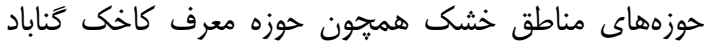
است.

\section{مواد و روشها منطقه موردمطالعه}

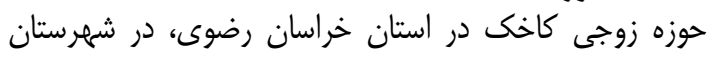

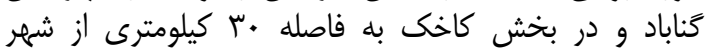

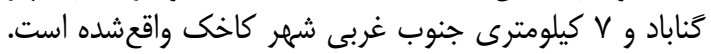

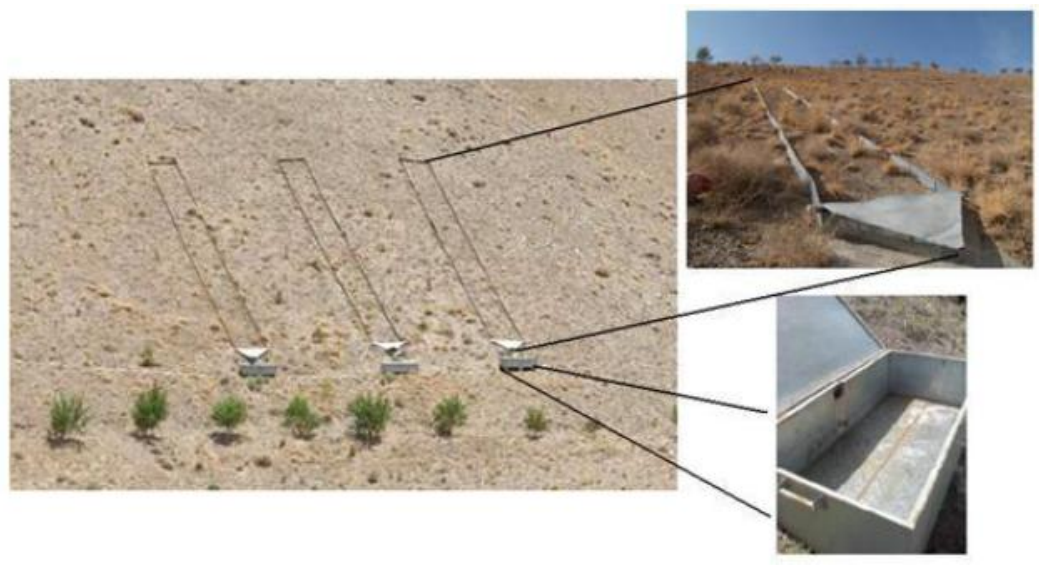

شكل ( - موقعيت جغرافيايى حوزه كاخك (نمونه و شاهد)

Figure1. Geographical location of Kakhk watershed (Sample and observation catchments)

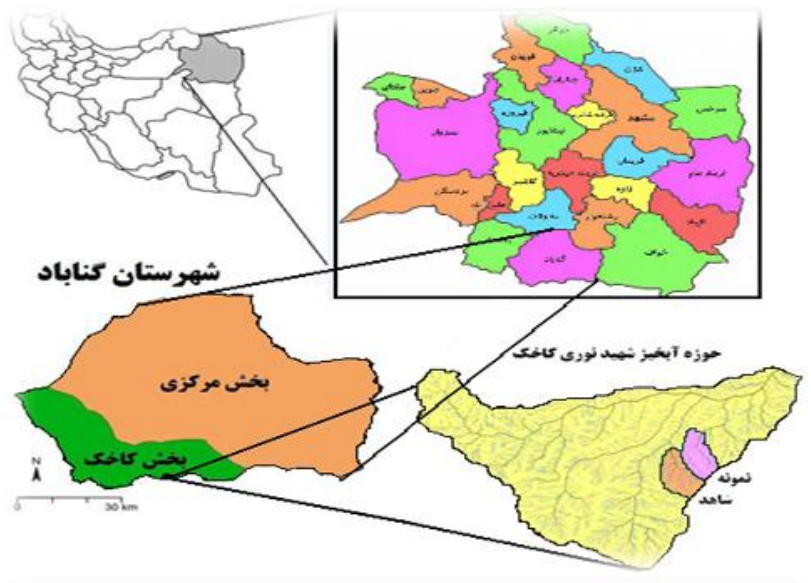

شكل r - نمونهاى از يلاتهاى فرسايشى در حوزه كاخى كناباد

Figure 2. Samples of erosion plots in Kakhk watershed of Gonabad 
اصلى رويكرد درخت تصميه، نشان دادن راهحلهاست (بَّ).



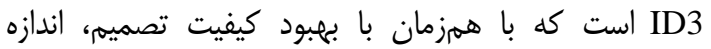



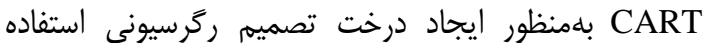

يار امتر هاى مورداستفاده در شبيلهسازى موريل

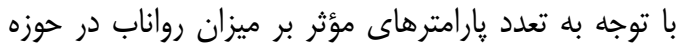

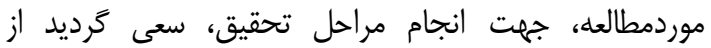

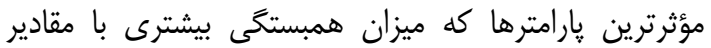

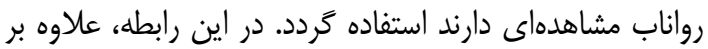

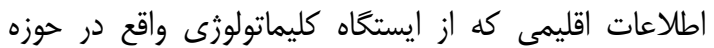

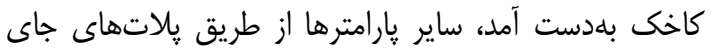

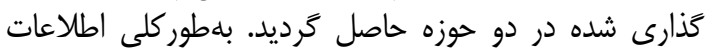

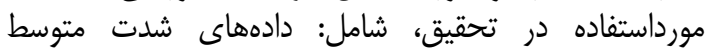

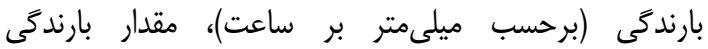
(ميلىمتر)، تداوم بارش (ساعت)، تبخير از تشتى (ميلى (ميلىمتر)،

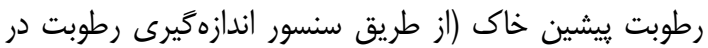

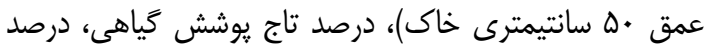

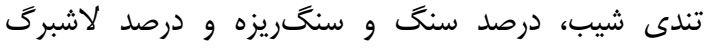
مىباشد. همجنين مقادير رواناب حاصل از بارش نئ نيز از از مخازن

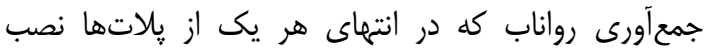

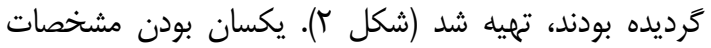

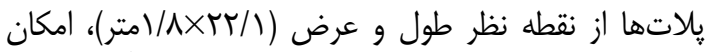

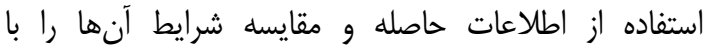

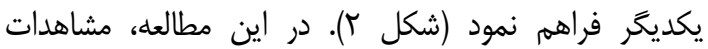

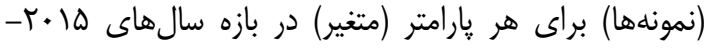

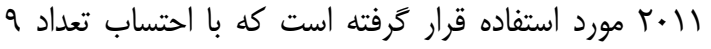

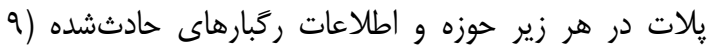
رَبار)، بالغ بر اي داده در هر حوزه ( بوا داده در حوزه زوجى)

با توجه به اختلاف در ماهيت و شيوهى اجراى هر هر يكى از

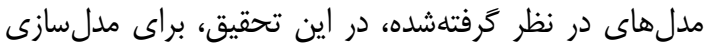

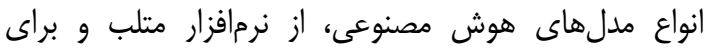

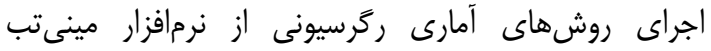

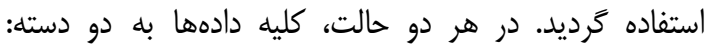

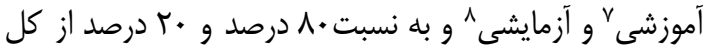

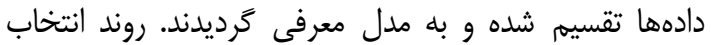

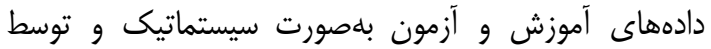

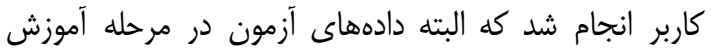

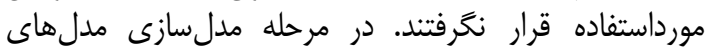

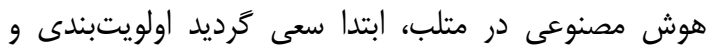

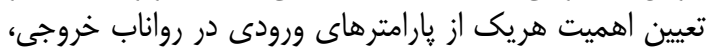

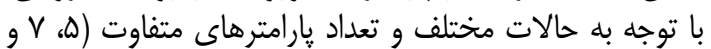

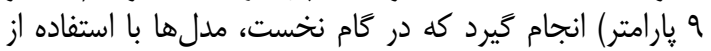

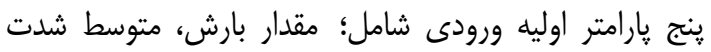

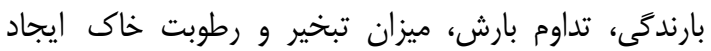

معرفى روش هاى مورد استفاده

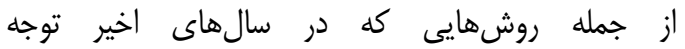

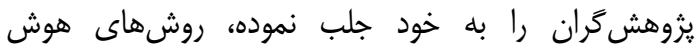

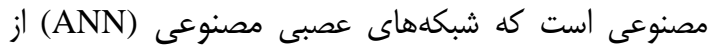

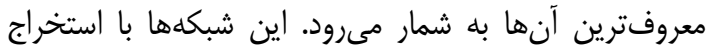



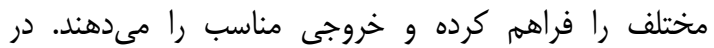

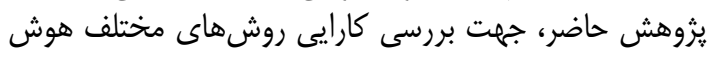

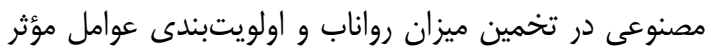

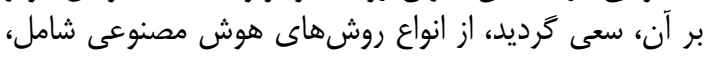

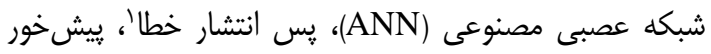

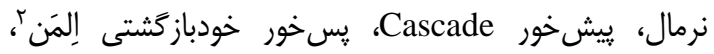

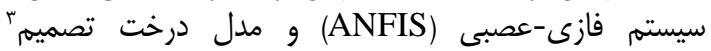

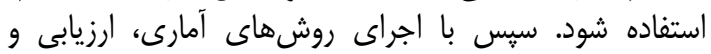

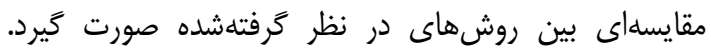

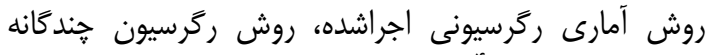

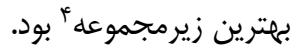

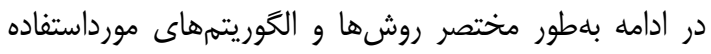
شرح داده مىشود.

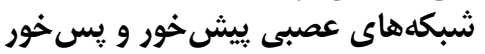

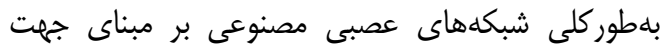

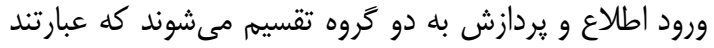

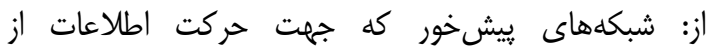

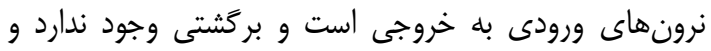

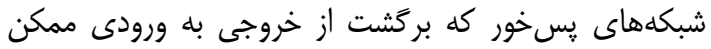

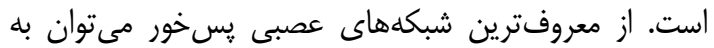

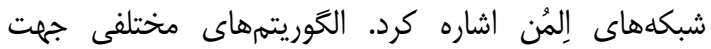

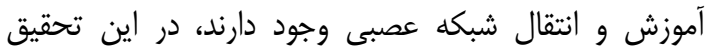

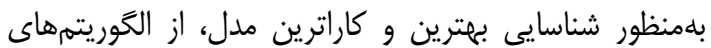

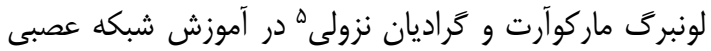

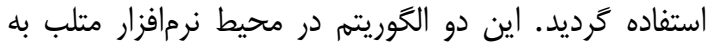



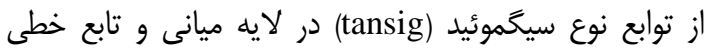
(purelin)



سيستم استنتاج فازى عصبى تطبيقى (ANFIS)

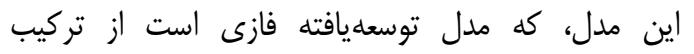

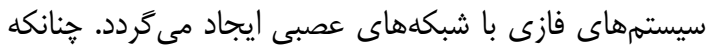

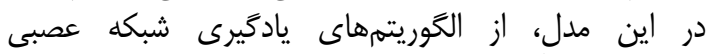

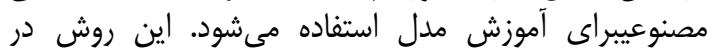

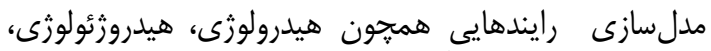

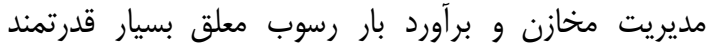

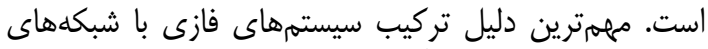
عصبى، قابليت يادَّيرى آنها است (19). درخت تصميم تصايت

درخت تصميم از انواع روشهاى دادهاوى و و ابزارى قوى

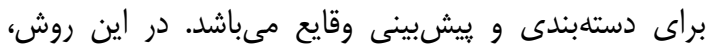

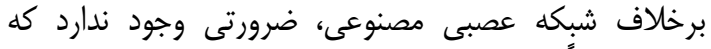

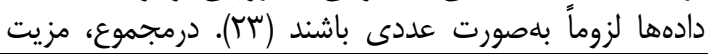
3- Decision Tree 4- Best subset 5- Gradient descent 7- Training data $\quad$ 8- Testing data 
كَياهى و درصد شيب هِلاتها و در كام آخر، مقادير بارامترهاى

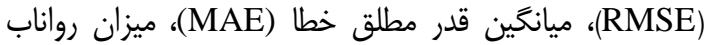

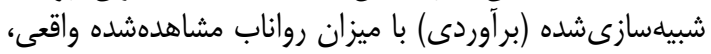
مقايسه و ميزان كارايى مدلها ها بررسى شدان رواب مثنا

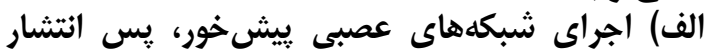
Cascade در اين حالت مدلهاى عصبى المتى تحت شرايط مختلف؛ تعداد

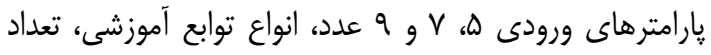

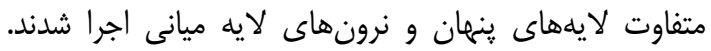

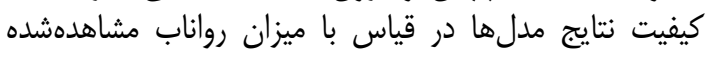



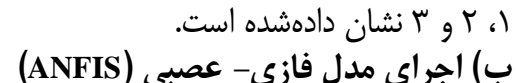

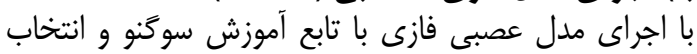

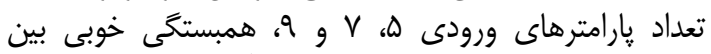

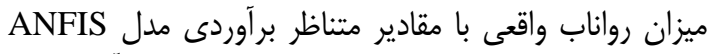

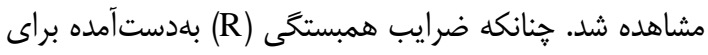

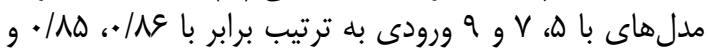

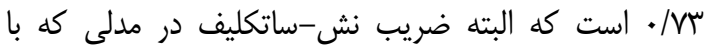

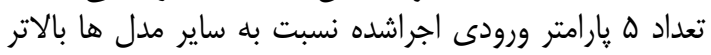

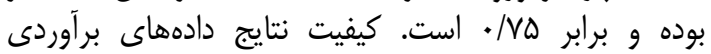
حاصل از مدل ANFIS در جدول ثأ ارائه شده است.
شدند و سبس در كام بعدى، پارامترهاى درصد تاج يوشش برائ

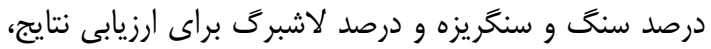
وارد مدلها كَرديدند.

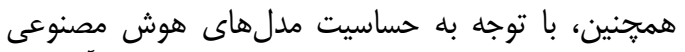

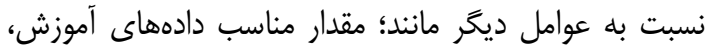

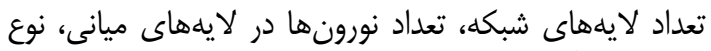

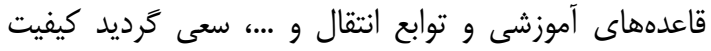

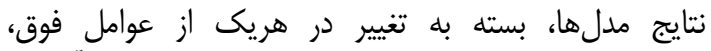
موردبررسى قرار كيرد. درنهايت با استفاده از دادههاى آزمون،

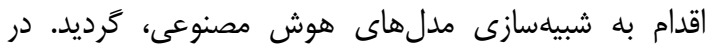

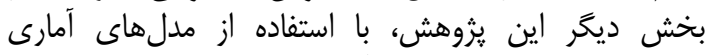

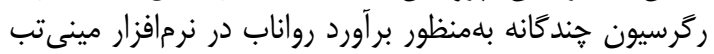

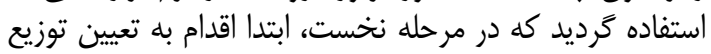

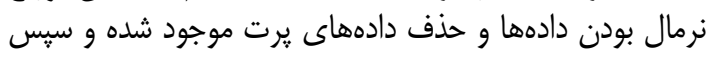

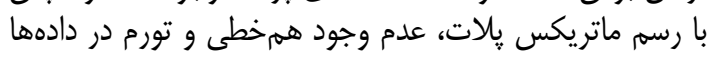



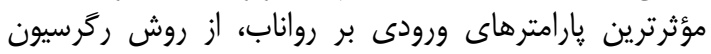

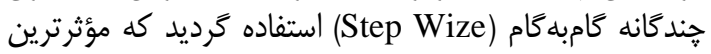

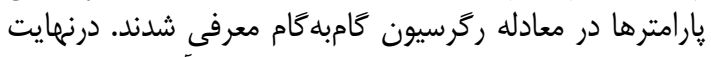

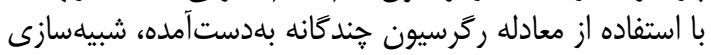

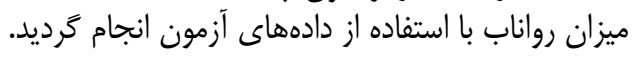

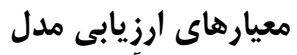

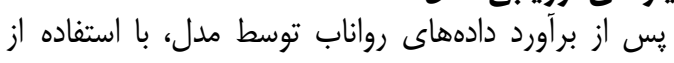

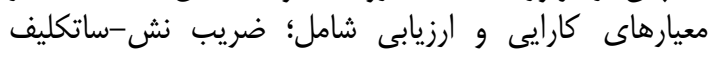

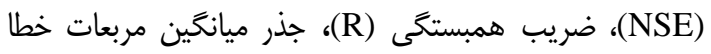


Feed forward جدول (- كيفيت نتايج مدل با استفاده از شبكه عصبى ييش خور نرمال Table 1. Quality of the results produced by the model using normal feed forward ANN

\begin{tabular}{|c|c|c|c|c|c|c|c|c|}
\hline تعداد يارامترهاى & تابع آموزش & (اول و دوم) & تعداد لايههاى & تعداد نرونهاى & $\mathrm{R}$ & $\begin{array}{c}\text { Nash, } \\
\text { Sutcliffe }\end{array}$ & RMSE & MAE \\
\hline$\Delta$ & trainlm & Tansig-purelin & 1 & $\Delta$ & $\cdot / \mathrm{MA}$ & $\cdot / \mathrm{H} \wedge$ & $T / 4$ & $1 / 9$ \\
\hline$\Delta$ & traingdx & Tansig-purelin & 1 & 1. & $\cdot / \mathrm{M}$ & $\cdot / V q$ & r & $1 / \Delta$ \\
\hline$\Delta$ & traingdx & Tansig-purelin & 1 & r. & $\cdot / \wedge \Delta$ & $\cdot / \mathrm{V}$ & $r / r$ & $1 / 9$ \\
\hline$\Delta$ & trainlm & Tansig-purelin & r & 1. & $\cdot / M$ & $\cdot / V \Delta$ & r & $1 / 8$ \\
\hline$\checkmark$ & trainlm & Tansig-purelin & 1 & 1. & $\cdot / \mathrm{VA}$ &.$|9|$ & $r / 9$ & 1/9 \\
\hline v & trainlm & Tansig-purelin & r & 1. & $\cdot / \mathrm{V} 9$ &.$/ 4$. & $r / 9$ & $1 / \mathrm{M}$ \\
\hline 9 & trainlm & Tansig-purelin & 1 & 1. & $\cdot / \Delta \Lambda$ & $\cdot / \cdot r$ & r & T/Ta \\
\hline
\end{tabular}

جدول r- كيفيت نتايج مدل با استفاده از شبكه عصبى يس انتشار CaNN Table 2. Quality of the results produced by the model using Cascade back propagation ANN

\begin{tabular}{|c|c|c|c|c|c|c|c|c|}
\hline تعداد يارامترهاى & تابع آموزش & تابع انتقال ( اول و & تعداد لايههاى & 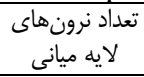 & $\mathrm{R}$ & $\begin{array}{c}\text { Nash, } \\
\text { Sutcliffe }\end{array}$ & RMSE & MAE \\
\hline$\Delta$ & trainlm & Tansig-purelin & 1 & 1. & $\cdot / \wedge \mathrm{V}$ & $V Q$. & $T / \cdot \Delta$ & $1 / \Delta$ \\
\hline$\checkmark$ & trainlm & Tansig-purelin & r & 1. & $\cdot|\Lambda|$ & .199 & T/ru & $1 / 89$ \\
\hline 9 & trainlm & Tansig-purelin & 1 & 1. & $.18 \mathrm{~V}$ & ./RT & $m / l$ & $r / T$ \\
\hline
\end{tabular}

جدول r - كيفيت نتايج مدل با استفاده از شبكه عصبى يس انتشار Elman Table 3. Quality of the results produced by the model using Elman back propagation ANN

\begin{tabular}{|c|c|c|c|c|c|c|c|c|}
\hline تعداد يارامترهاى & تابع آموزش & تابع انتقال ( اول و دوم) & تعداد لايههاى & تعداد نرونهاى & $\mathrm{R}$ & $\begin{array}{c}\text { Nash, } \\
\text { Sutcliffe }\end{array}$ & RMSE & MAE \\
\hline$\Delta$ & trainlm & Tansig-purelin & 1 & 1. & $\cdot / \mathcal{H}$ & .199 & $t / \mu$ & $1 / 99$ \\
\hline$\checkmark$ & trainlm & Tansig-purelin & 1 & 1. & $\cdot / \Lambda \Delta$ & $\cdot / \mathrm{VT}$ & $T / / Q$ & $1 / \mathrm{N}$ \\
\hline 9 & trainlm & Tansig-purelin & 1 & 1. & $\cdot / N^{\mu}$ & $\cdot / t^{2}$ & $r / \cdot V$ & $r / T \Delta$ \\
\hline
\end{tabular}

جدول ع- كيفيت نتايج مدل با استفاده از شبكه فازى- عصبى (ANFIS) Table 4. Quality of the results produced by the model using Adaptive Neuro-Fuzzy Inference

\begin{tabular}{|c|c|c|c|c|c|}
\hline تعداديارامترهاى ورودى & تابع آموزش & $\mathrm{R}$ & Nash, Sutcliffe & RMSE & MAE \\
\hline$D$ & sugeno & $\cdot / \wedge g$ & $\cdot / v \Delta$ & $\Gamma / \Gamma \wedge$ & $1 / 99$ \\
\hline$v$ & sugeno & $\cdot / \wedge \Delta$ & ع & $r / / Q$ & $1 / v 1$ \\
\hline 9 & sugeno &.$/ v$ & $\cdot|\theta|$ & $r / \cdot \gamma$ & $r / r \Delta$ \\
\hline
\end{tabular}

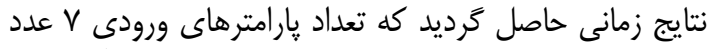



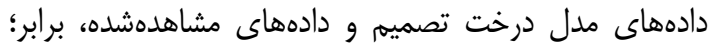

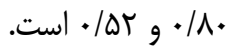

ج) اجراى مدل درخت تصميم (Decision Tree)

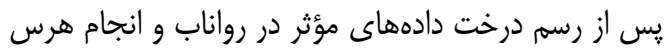

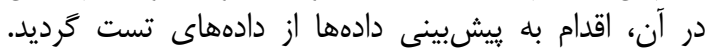
همانطور كه در جدول ه نمايش داده داده شده است، بهترين

جدولهـ- كيفيت نتايج مدل با استفاده از مدل درخت تصميم ركرسيونى Regression Tree Table 5. Quality of the results produced by the model using Regression Tree

\begin{tabular}{|c|c|c|c|c|c|}
\hline تعداد يارامترهاى ورودى & الخوريته مورداستفاده & $\mathrm{R}$ & Nash, Sutcliffe & RMSE & MAE \\
\hline$\Delta$ & CART & $\cdot / V \Delta$ & $\cdot / V \Delta$ & $\Delta / \cdot 1$ & $T / T \Delta$ \\
\hline$\checkmark$ & CART & $\cdot / \Lambda$ & $\cdot / \Delta T$ & 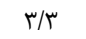 & $r / s$ \\
\hline 9 & CART & $\cdot / V \Delta$ & $\cdot / \Delta T^{C}$ & $r / V \Lambda$ & $1 / V$ \\
\hline
\end{tabular}

گرديد، كه از بين 9 يارامتر در نظر گرفتهشده، يارامترهاى

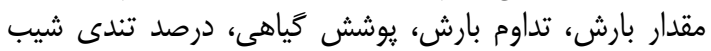

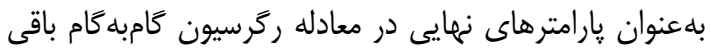

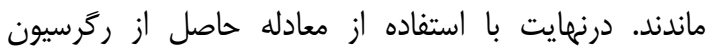

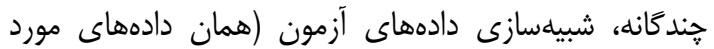

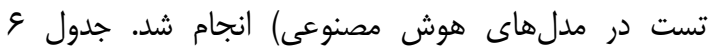

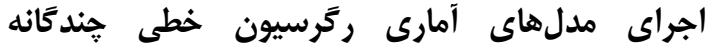
(Multiple Regression )

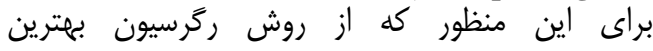



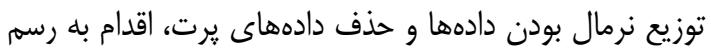

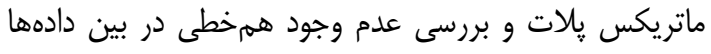


مشاهدهاى مىباشد. همجنين در شكل بّا، نمودار ارتباط


مشاهدهشده واقعى به نمايش كذاشت مدادهده است.

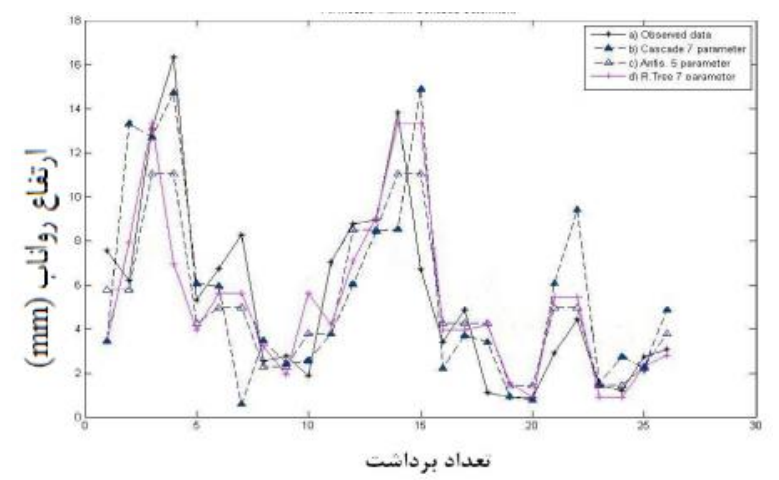

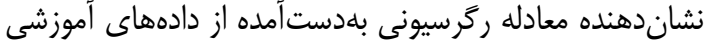

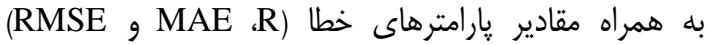
مربوط به مقايسه برأورد مدل ركرّسيونى هندمتئيره و مقادير

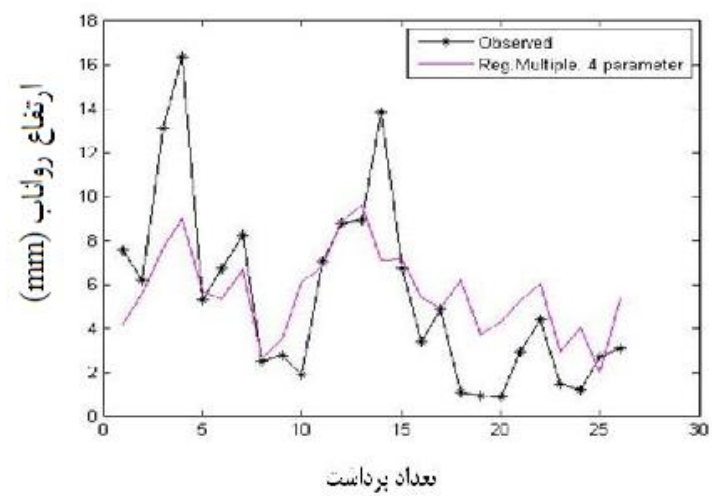



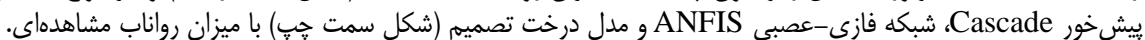

Figure 3. Comparison of the predictions produced by Stepwise Multiple Regression model and the measured data (right) and also the results produced by different Cascade feed forward ANN structures, ANFIS and the decision Tree models against measured values of run off (left).

جدول צ- كيفيت نتايج حاصل از مدل ركرسيون جندكانه گامبه گام Step Wise Multiple Regression

\begin{tabular}{|c|c|c|c|c|c|}
\hline تعداد يارامترهاى ورودى & روشهاى مورداستفاده & $\mathrm{R}$ & Nash, Sutcliffe & RMSE & MAE \\
\hline r & Best subset -Step Wise & $\cdot / \mathrm{NT}$ & . & $r / \cdot r$ & T/r \\
\hline \multicolumn{3}{|c|}{$\mathrm{Y}=0.52+0.0805 \mathrm{x}_{1}-0.154 \mathrm{x}_{3}-0.038 \mathrm{x}_{6}+0.0897 \mathrm{x}_{7}$} & \multicolumn{3}{|c|}{ معادله ركر سيون بهدستآمده: } \\
\hline
\end{tabular}

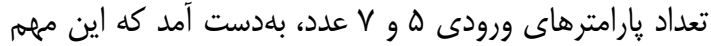



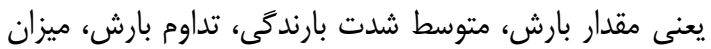

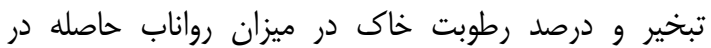

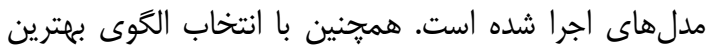

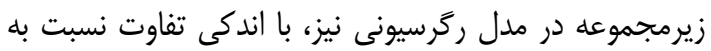

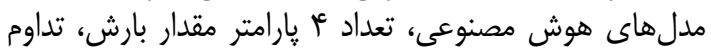

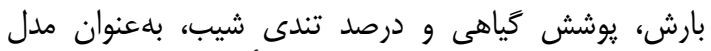

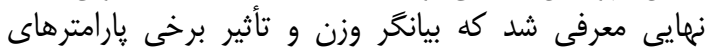

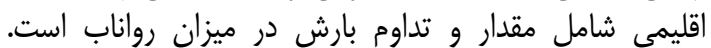

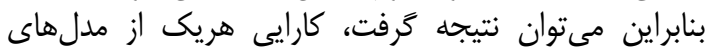

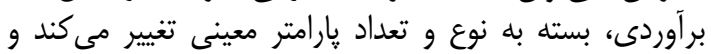

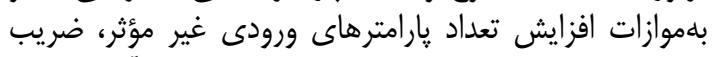

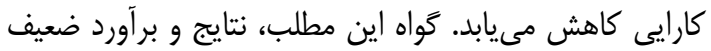

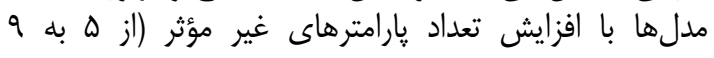
يارامتر) است.

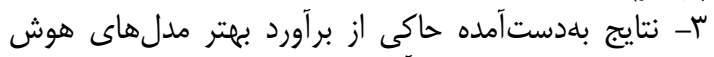

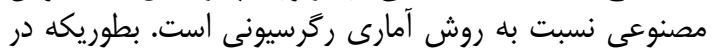



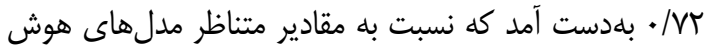


(IV)
با توجه به بررسىهاى انجامشده در تحقيق، نتايج زير حاصل مى گرد درد: 1- همانطور كه از نتايج مشخص است؛ عملكرد روشهاى

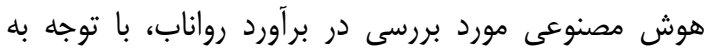

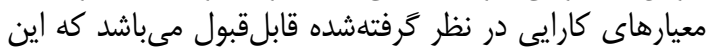

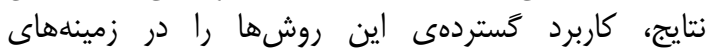



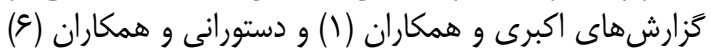

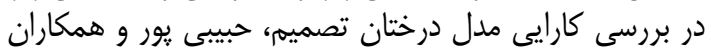



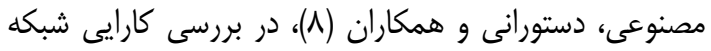



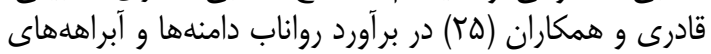

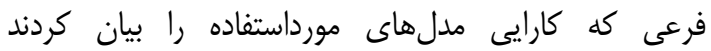
همخوانى دارد.

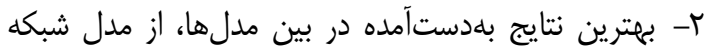

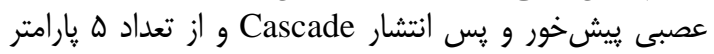

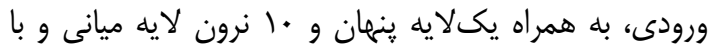

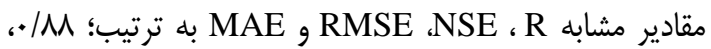

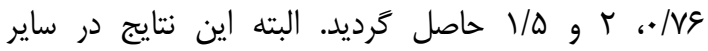


إلمَن و سيستم فازى عصبى ANFIS و درخت تصميهم) نيز با 
f- همجنين نتايج نشان داد شبكه عصبى مصنوعى كارايى

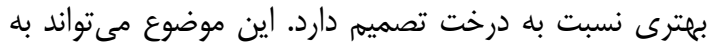

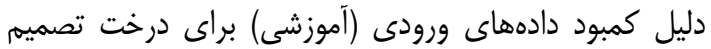



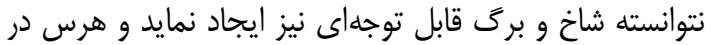

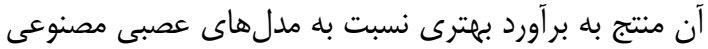

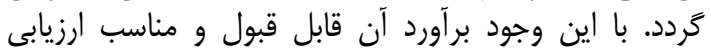

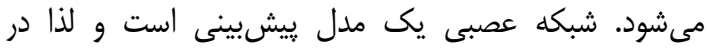

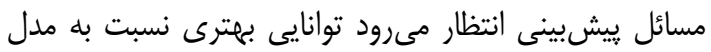

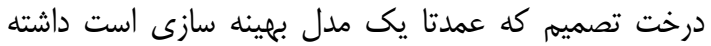

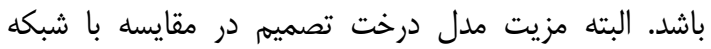

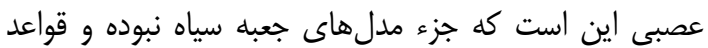

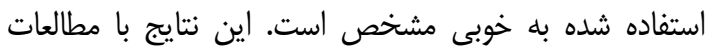

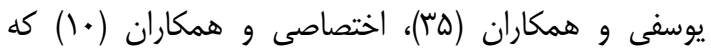

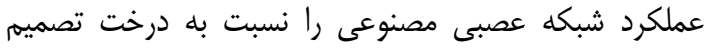
بالاتر دانسته، مطابقت دارد و با نتايج سنتيل كومار وار و همكاران

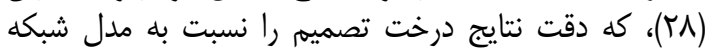

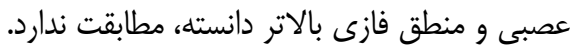

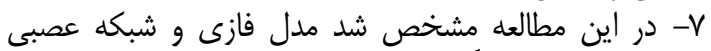

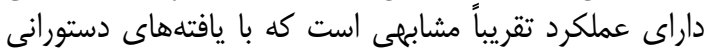

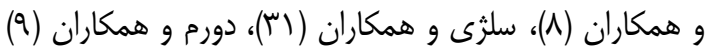

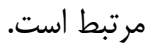
ج- همانطور كه در نمودارهاى حاصل از شبكههاى عصبى إنى

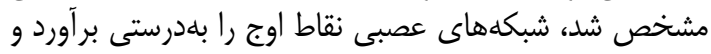



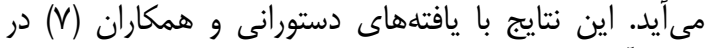

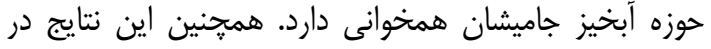



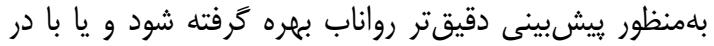

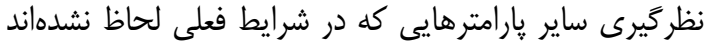

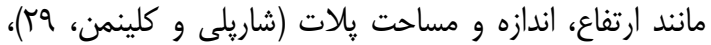

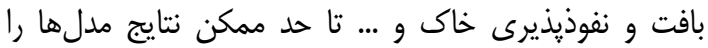

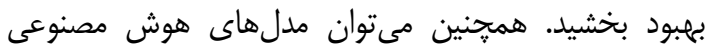

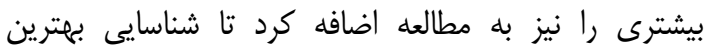
مدلها به صورت جامع ترى صورت كيرد.

لازم است از اداره منابع طبيعى و آبخيزدارى شهرئرستان

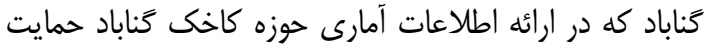

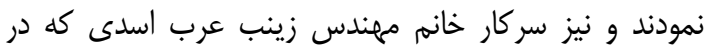

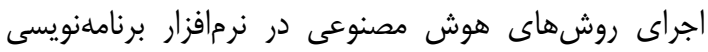
MATLAB راهنمايى كردند تقدير و تشكر 5 رددد.
مصنوعى، باتاجاريا و همكاران (أ) در مدلسازى بار رودخانه

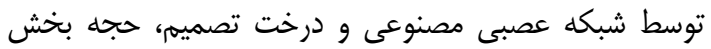

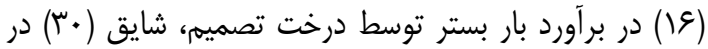

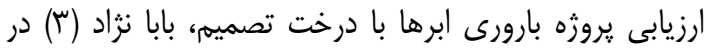

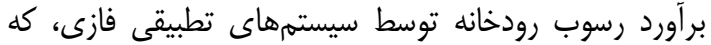

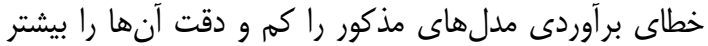

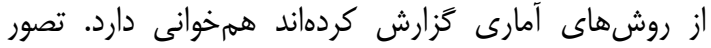

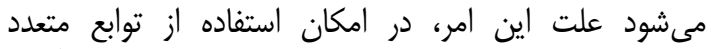

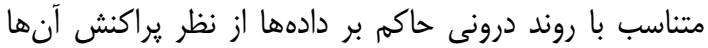

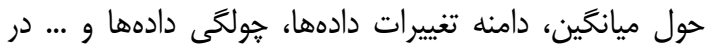

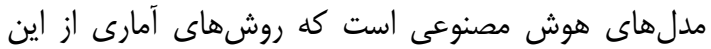

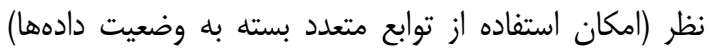

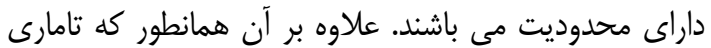

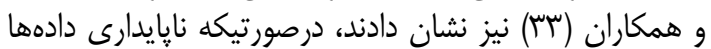

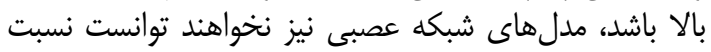

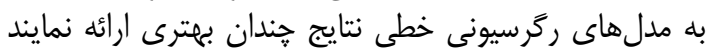



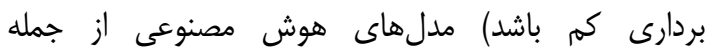

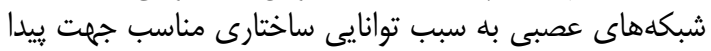

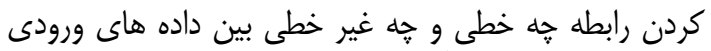

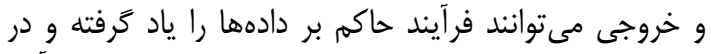

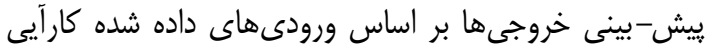

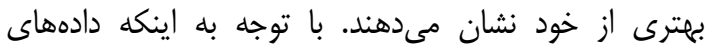

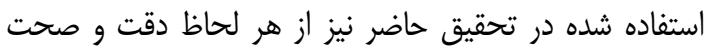

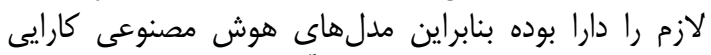

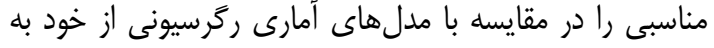

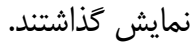

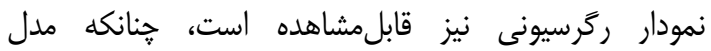

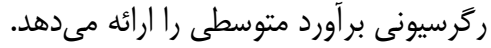

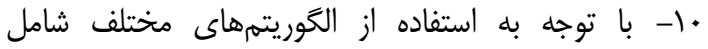

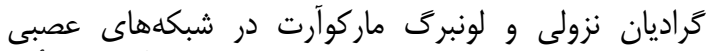

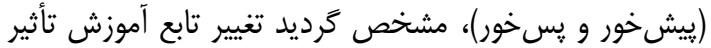
جندانى در كيفيت نتايج نمايى ندارد.

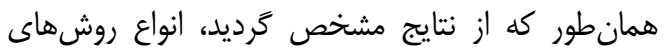

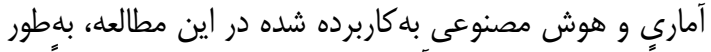

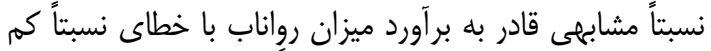



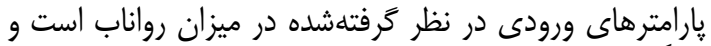

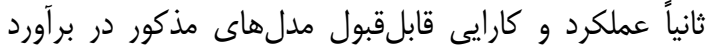

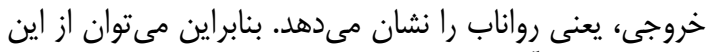



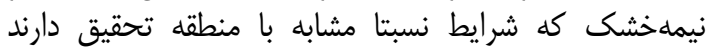

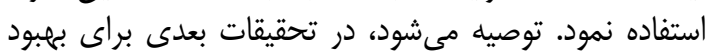



classification on accurately suspended sediment estimation using decision tree models. The first national conference on achieving sustainable development in agriculture, natural resources and environment sectors, Tehran (In Persian).

2. Asadi, S., J. Shahrabi, P. Abbaszadeh and S. Tabanmehr. 2013. A new hybrid artificial neural networks for rainfall-runoff process modeling. Neurocomputing, 121: 470-480.

3. Babanejad, T. 2012. The use of genetic algorithms in adaptive fuzzy-neural system to estimate the sediment in the river, Ninth International River Engineering Seminar, Shahid Chamran University of Ahvaz, Iran, February 2012, (In Persian).

4. Bhattacharya, B., R.K. Price and D.P. Solomatine. 2007. Machine learning approach to modeling sediment transport. Journal of Hydraulic Engineering. 133(4): 440-450.

5. Chen, J.C., S.K. Ning, H.W. Chen and C.S. Shu. 2008. Flooding probability of urban area estimated by decision tree and artificial neural networks, Journal of Hydroinformatics, 10(1): 57-67.

6. Dastorani, M.T., A. Habibpour, M.R. Ekhtesasi, A. Talebi and J. Mahjubi, 2013. Evaluation of the Decision Tree Model in Precipitation Prediction (Case study: Yazd Synoptic Station), Iran Water Resources Research, 3: 14-27 (In Persian).

7. Dastorani M.T., Kh. Azimi Fashi, A. Talebi and M.R. Ekhtesasi, 2012. Estimation of Suspended Sediment Using Artificial Neural Network (Case Study: Jamishan Watershed in Kermanshah). Journal of watershed management research, 6: 61-74 (In Persian).

8. Dastorani M.T., H. Sharifi Darani, A. Talebi and A.R. Moghaddamnia, 2011. "Evaluation of the application of artificial neural networks and adaptive neuro-fuzzy inference systems for rainfall-runoff modeling in Zayandeh_rood dam basin, Journal of Water and Wastewater, 4: 114-125 (In Persian).

9. Dorum, A., A. Yarar, M. Faik Sevimli and M. Onüçyildiz. 2010. "Modelling the rainfall-runoff data of susurluk basin." Expert Systems with Applications 37(9): 6587-6593.

10. Ekhtessasi, M., M. Yusefi and M. Tavakkoli. 2015. Comparing the Best Input Combining Artificial Neural Networks and Decision Tree Method to Identify Factors that Influence the Phenomenon of Dust Storm (Case Study Yazd Province), Journal of Watershed Science and Engineering. 9(28): 33-40 (In Persian).

11. Eshghi, P., J. Farzadmehr, M.T. Dastorani and Z. Arab Asadi. 2016. The effectiveness of intelligent models in estimating the river suspended sediments (Case study: Babaaman basin, Northern Khorasan), Journal of watershed management research, 14: 88-95 (In Persian).

12. Eshghizadeh, M. 2011, The Review of the pair watersheds of Kakhk, Gonabad, Department of Natural Resources and Watershed Management of Gonabad (In Persian)

13. Fathollahi, S., D. Mirshahi and B. Abbaspoor, 2015. Prediction of runoff resulted from rainfall in Ajichai river basin, using neural networks. The first International Congress on Irrigation and Drainage, Ferdowsi University of Mashhad. Iran (In Persian).

14. Goswami, M. and K.M. O'Connor, 2005. Application of Artificial Neural Networks for river flow simulation in three French catchments. $4^{\text {th }}$ Inter-Celtic Colloquium on Hydrology and Management of Water Resource. National University of Ireland, Galway, Ireland.

15. Habibipour, A., M.T. Dastorani, M.R. Ekhtesasi, H. Afkhami. 2011. Evaluation of the Effects of Data range Modification on Efficiency of Regression Decision Tree and Artificial Neural Networks for Drought Prediction, Journal of watershed management research, 3: 63-79 (In Persian).

16. Hajjahbakhsh, C. 2012. Estimation of bed load sediment using regression decision trees, and comparing with experimental methods, M.Sc. Thesis, Department of Civil Engineering, Yazd University, Iran, 103 pp (In Persian).

17. Hamzaçebi, C. 2008. Improving artificial neural network's performance in seasonal time series forecasting. Information Sciences, 178(23): 4550-4559.

18. Hung, N.Q., M.S. Babel, S. Weesakul and N.K. Tripathi. 2008. An articial neural network model for rainfall forecasting in Bangkok, Thailand. Hydrology and Earth System Sciences Discussions, 5(1): $183-218$

19. Jang, J.S.R. 1993. ANFIS: Adaptive-network-based fuzzy inference system. J. of IEEE. Trans. Syst. Man, Cyber, 23(3): 665-685.

20. Janikow. Z. and M. Faifer. 2000. Bottom- un Fuzzv Partitioning in Fuzzv Decision Trees. Fuzzy Information Processing Society, NAFIPS. $19^{\text {th }}$ International Conference of the North American.

21. Jeong, C.S., W.J. Koh and J.H. Heo. 2000. A study on real-time forecasting of reservoir inflow based on artificial neural network. Proceedings of Watershed Management and Operations Management 2000 conference, American Society of Civil Fngineers, USA

22. Karamouz, M. and S. Araghinejad. 2011. Advanced Hydrology. Amirkabir University of Technology Press. Tehran. Iran. (In Persian).

23. Kia, M. 2010. Neural networks in MATLAB, Third Edition, published by Kian-Rayaneh-Sabz, 229 pp (In Persian).

24. Meshkani, A. and A. Nazemi. 2009. Introduction to Data Mining, Ferdowsi University of Mashhad Press, Mashhad (In Persian).

25. Nourani, V., Ö. Kisi and M. Komasi. 2011. "Two hybrid Artificial Intelligence approaches for modeling rainfall-runoff process." Journal of Hydrology 402(1-2): 41-59.

26. Nourani, V., M.A. Kynejad and L. Malekani. 2010. "The use of Adaptive Neural - fuzzy systems in Modeling of rainfall - runoff." Journal of Civil and Environmental Engineering, University of Tabriz 39(4): 75-81 (In Persian). 
27. Qaderi, M. 2012. Development of hydrological model for simulation of rainfall-runoff from hill slopes and small catchments (Case study: Sanganeh station, Khorasan Razavi), M.Sc. Thesis, Faculty of Natural Resources, Yazd University, Iran (In Persian).

28. Senthil Kumar, A., C. Ojha, M. Goyal, R. Singh and P. Swamee. 2012. "Modeling of Suspended Sediment Concentration at Kasol in India Using ANN, Fuzzy Logic and Decision Tree Algorithms". Journal of Hydrologic Engineering, 17(3): 393-404.

29. Sharpley, A. and P. Kleinman. 2003. Effect of rainfall simulator and plot scale on overland flow and phosphorustransport. Journal of Environtal Quality, 32: 2172-2179

30. Shayegh, M. 2012. Cloud seeding project evaluation using decision trees regression model (case study: in central Iran, Fars province), MSc thesis, Water and Power Industry University, Tehran. Iran. (In Persian).

31. Solgi, A., F. Radmanesh, A. Pourhaghi and M. Bagherian. 2014. Evaluation of Artificial Intelligence Systems Performance in Precipitation Forecasting. TI Journals Agriculture Science Developments. 3 (7): 256-264.

32. Talei, A., L.H.C. Chua, C. Quek and P.E. Jansson. 2013."Runoff forecasting using a Takagi-Sugeno neuro-fuzzy model with online learning." Journal of Hydrology, 488(0): 17-32.

33. Tamari, S., J.H.M. Wosten and J.C. Ruz-suarez. 1996. Testing an artificial neural network -for predicting soil hydraulic conductivity. Journal of Soil Science Society of America, 60: 1732-1741.

34. Vahabi, J. and M.H. Mahdian. 2008. Rainfall simulation for the study of the effects of efficient factors on runoff rate. Current Science, 95: 1439-1445.

35. Yousefi, M. and F. Barzegar, 2013. Suspended sediment comparative study using a decision tree model and sediment curve (Case Study: Lorestan) Journal of Watershed management Research (Pajoohesh and Sazandegi), in press (In Persian). 


\title{
Evaluation of the Efficiency of Different Artificial Intelligence and Statistical Methods in Estimating the Amount of Runoff (Case Study: Shahid Noori Watershed of Kakhk, Gonabad)
}

\author{
Mohammad Mahdi Zarei ${ }^{1}$, Mohammad Taghi Dastorani ${ }^{2}$, Mansour Mesdaghi ${ }^{3}$ and \\ Masoud Eshghizadeh ${ }^{4}$ \\ 1 and 3- M.Sc. Graduate in Watershed Management and Visiting Professor, Faculty of Natural Resources and \\ Environment, Ferdowsi University of Mashhad \\ 2- Professor, Faculty of Natural Resources and Environment, Ferdowsi University of Mashhad, Iran \\ (Corresponding author: dastorani@um.ac.ir) \\ 4- Assistant Professor, Department of Agriculture and Natural Resources, University of Gonabad \\ Received: December 26, 2015 Accepted: September 4, 2016
}

\begin{abstract}
Rainfall-runoff models are used in the field of hydrology and runoff estimation for many years, but despite existing numerous models, the regular release of new models shows that there is still not a model that can provide sophisticated estimations with high accuracy and performance. In order to achieve the best results, modeling and identification of factors affecting the output of the model is necessary. In this regard, in present study, it has been tried to identify the factors and estimating the amount of runoff using a variety of methods of artificial intelligence and multiple regression. Then, to evaluate the efficiency of the implemented models and choose the best model, some performance criteria including the correlation coefficient (R), Nash-Sutcliffe coefficient (NSE), the root mean square error (RMSE) and the mean absolute error (MAE) were used. The data used in this study were 9 rainfall events data measured in time period of 2011- 2015 taken from the Khakh watershed of Gonabad. Artificial intelligence models used in this study were: normal feedforward neural networks, feedforward Cascade neural networks, feedbackward Elman neural networks, Adaptive Neuro Fuzzy Inference System (ANFIS) and regression decision tree model (Regerssion Tree) that were implemented in MATLAB software environment and also step multiple regression as statistical methods which was implemented in Minitab software. The results of this study showed that the used statistical and artificial intelligence methods are considered acceptable with almost similar performance and with relatively appropriate accuracy and low error they are able to estimate the amount of runoff. In the meantime, Cascade and normal feedforward neural models with 5 input parameters, presented better performance comparing to the other models, as the performance criteria of R, RMSE, NSE and MAE in these models were the similar values of $0.88,0.76,2$ and 1.5 , respectively. Overall, the findings indicate better estimations of the artificial intelligence models comparing to the regression model.
\end{abstract}

Keywords: Modeling, Neural Networks, Decision trees, ANFIS, Backpropagation, Feedforward, Feedbackward 